\title{
Effect of Metal Cations on the Kinetics of Formation and Structure of Gels Formed during the Acid Hydrolysis of Tetraethoxysilane
}

\author{
E. A. Tarasenko ${ }^{a}$, O. E. Lebedeva ${ }^{a, *}$, G. S. Peters ${ }^{b}$, and A. A. Veligzhanin ${ }^{b}$ \\ ${ }^{a}$ Belgorod State National Research University, Belgorod, 308015 Russia \\ ${ }^{b}$ National Research Center Kurchatov Institute, Moscow, 123098 Russia \\ *e-mail: OLebedeva@bsu.edu.ru \\ Received November 6, 2018; revised February 11, 2019; accepted February 12, 2019
}

\begin{abstract}
The hydrolysis and polycondensation of tetraethoxysilane are studied at low pH values in the presence of doping additives (cations of various metals). The period of gel formation is found via viscometry, and the activation energy is calculated. It is shown that the doping additives affect both of these parameters. A change in particle size during the hydrolytic polycondensation of tetraethoxysilane in the presence of metal cations is studied via dynamic light scattering. The structural characteristics of the gels-the radius of gyration and the fractal dimension of the surface-are found through small-angle X-ray scattering. A mechanism of particle formation-kinetically controllable aggregation of a cluster-cluster type-is proposed.
\end{abstract}

Keywords: hydrolysis of tetraethoxysilane, metal cations, gelation, activation energy, fractal dimension

DOI: $10.1134 / \mathrm{S} 0036024419090279$

\section{INTRODUCTION}

The sol-gel process is a promising way of obtaining new materials with desired properties. It enables us to produce different inorganic materials, e.g., glass, ceramics, aerogels, films, and powdered hybrid materials with adjustable microstructure and properties [1-4].

Tetraethyl ester of orthosilicic acid (tetraethoxysilane or TEOS) is often used as a precursor in the solgel production of silicate materials. It is possible to obtain various materials via its hydrolysis with subsequent polycondensation [5-8], where the dopants are introduced directly into the initial solution. The $\mathrm{pH}$ value and the hydrolysis catalyst strongly affect the structure of the final product. Mineral acids (e.g., hydrochloric and nitric acid) are often used as catalysts for acid hydrolysis, while ammonium hydroxide is used for basic hydrolysis. Acetic acid, potassium hydroxide, amines, potassium fluoride, hydrofluoric acid, titanium alkoxides, vanadium alkoxides and oxides are less common catalysts for hydrolysis [9-11].

There are three ranges of $\mathrm{pH}$ values, where hydrolysis and polycondensation display their own characteristic features: $\mathrm{pH}<2, \mathrm{pH}$ within $2-7$, and $\mathrm{pH}>7$. If hydrolysis proceeds within $\mathrm{pH} 2-7$, branched structures and three-dimensional networks of gels are formed. Large secondary particles with shapes close to spherical are formed in the alkaline environment [12]. The range of $\mathrm{pH}<2$ is metastable. It is believed that the silica sol particles have a positive charge in this range of $\mathrm{pH}$, and the rate of polymerization is propor- tional to the concentration of protons [12]. The gel structure forms in several hours at low $\mathrm{pH}$ values, while this takes several days at other $\mathrm{pH}$ values.

Electrolytes also have an appreciable effect on the formation of gel structures. There are data on the mechanisms behind the influence metal cations with different charges have on the coagulation of silica sols. Most of these are based on the assumption that cations can act as agents that form bridge bonds between sol particles, due to the adsorption of cations on the surfaces of silica sol. These results, however, were obtained at $\mathrm{pH} 4.5-11$ [13]. The range of hydrolysis of TEOS at low $\mathrm{pH}$ values is therefore the one least studied, and there are no data on the effect cations have on the synthesis of silicate materials and their structure in this range.

The aim of this work was to study the hydrolysis of TEOS in the metastable region in the presence of metal cations leading to the production of new functional silicate materials, and to study the structure of these materials.

\section{EXPERIMENTAL}

Chemically pure TEOS $\left(\mathrm{Si}\left(\mathrm{C}_{2} \mathrm{H}_{5} \mathrm{O}\right)_{4}\right)$ was used as a precursor for the preparation of silicate materials, while metal cations with different charges were used as additives. These included silver, sodium, and potassium nitrates, along with crystalline hydrates of nickel(II), cobalt(II), iron(III), and cerium(III) nitrates. The gels were prepared via the hydrolysis of a 


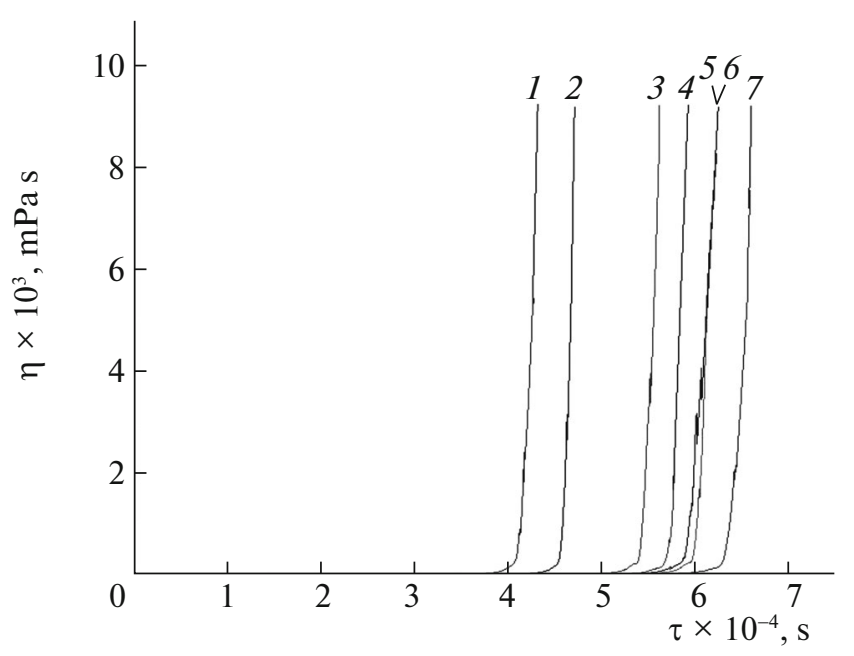

Fig. 1. Dependences of solution viscosity on TEOS hydrolysis time at $40^{\circ} \mathrm{C}$ in the presence of metal cations: (1) $\mathrm{Ni}^{2+}$, (2) without additives, (3) $\mathrm{Ce}^{3+}$, (4) $\mathrm{Fe}^{3+}$, (5) $\mathrm{Ag}^{+}$, (6) $\mathrm{Co}^{2+}$, and (7) $\mathrm{Na}^{+}$.

water-alcohol solution of TEOS in an acidic environment using nitric acid as a catalyst. A metal salt sample was dissolved in a given volume of distilled water. The weight of the sample was calculated such that the amount of metal cations was $3 \times 10^{-4}$ mol per $1 \mathrm{~g}$ of dry $\mathrm{SiO}_{2}$ powder obtained after the calcination of xerogel. The required volumes of TEOS, ethanol, and nitric acid were added to the salt solution. As a result, transparent colorless or faintly colored solutions with a TEOS : ethanol: $\mathrm{H}_{2} \mathrm{O}: \mathrm{HNO}_{3}=1: 4: 16: 0.6$ molar ratio were obtained. The resulting solutions were thermostatted until a stable gel formed.

The kinematic viscosity of silica sol during the process was recorded on a SV-10 vibration viscometer (Japan) up to $10000 \mathrm{mPa}$. The viscosity of the solutions was measured at 40,50 , and $60^{\circ} \mathrm{C}$ to calculate activation energy of gelation. The change in hydrodynamic particle size was studied via dynamic light scattering on a ZetaSizer Nano ZS analyzer (Malvern Instruments, United Kingdom) equipped with a $\mathrm{He} / \mathrm{Ne} 633 \mathrm{~nm}$ laser. The structure of the final polycondensation products (gels) was studied by means of small-angle X-ray scattering (SAXS). The measurements were made at the BioMUR small-angle X-ray scattering station of the Kurchatov synchrotron radiation source using a Pilatus $31 \mathrm{M}$ detector (Dectris) in the scattering vector range of $0.04-1.7 \mathrm{~nm}^{-1}$. A singlecrystal $\mathrm{Si}(111)$ focusing monochromator was employed at a wavelength of $0.1445 \mathrm{~nm}$. The structure of the gels was described in terms of radius of gyration $R_{\mathrm{g}}$ and fractal dimensions.

\section{RESULTS AND DISCUSSION}

The hydrolysis of TEOS proceeds at a fast rate [12], and polycondensation begins in the first minutes of the experiment, so the moment all components are put into the reaction mixture is considered to be the start of gelation. Figure 1 shows the curves of viscosity change over time at $40^{\circ} \mathrm{C}$ for silica sols doped with different cations.

The change in the viscosity of all sols is similar and has three time intervals. At the first stage, the viscosity of the sols increases monotonically and very slowly, testifying to the stability of the sol-gel systems. A stable state for silica gel without the addition of doping cations is observed for $\sim 11 \mathrm{~h}$, and the viscosity during this period varies from 2.21 to $10.00 \mathrm{mPa}$ s. The second time period then appears, during which there is a sharp rise in viscosity, resulting in a sharp drop in the turnover of sols and the formation of a spatial gel network. The time in which there is an intense increase in viscosity is the period of gelation; it characterizes the transition of the sol to the gel, along with the formation and compaction of the three-dimensional gel network. The time at which the viscosity of the solution reaches $400 \mathrm{mPa} \mathrm{s}$ is conventionally considered the end of gelation for all samples. At the third stage, the gel structure is compacted because of the formation of additional bonds between the structural elements of the net, which further increases the viscosity of the system.

Introducing doping metal cations into the initial system normally extends the sol's lifetime considerably, which lengthens the period of stabilization. The longest stable state is observed for silica gel doped with sodium cations $(\sim 15.8 \mathrm{~h})$. There are, however, some exceptions for cations. Indeed, the period of sol stability at $40^{\circ} \mathrm{C}$ is $9.8 \mathrm{~h}$ when using nickel cations, which is $110 \%$ shorter than with pure silica gel. The time needed to reach gelation at $40^{\circ} \mathrm{C}$ increases in the series $\mathrm{Ni}^{2+}<$ silica gel without alloying additives $<\mathrm{Ce}^{3+}<$ $\mathrm{Ag}^{+}<\mathrm{Fe}^{3+}<\mathrm{Co}^{2+}<\mathrm{Na}^{+}$. The expansion of the region of sol existence, which precedes the formation of a gel network when metal ions are introduced into the solution, indicates that the cations participate in the hydrolysis and polycondensation of TEOS.

The activation energies were calculated from the data obtained during the rheological studies of gelation at different temperatures. The calculations were performed using the Arrhenius equation. The average rate of gelation was the reverse period of gelation:

$$
\ln \left(t_{\mathrm{gel}}\right)=\ln A^{\prime}+e^{\frac{E_{G}}{R T}}
$$

The calculated activation energies for gelation are given in Table 1. The results indicate that metal ions affect not only the period of gelation, but also its activation energy. The activation energy for gelation can be higher or lower than the one calculated for pure sil- 
ica gel. There is no correlation between the activation energy and the charge of cations.

The data on the distribution of hydrodynamic particle sizes over the volume at different stages of the condensation of gels obtained via dynamic light scattering confirm that metal cations affect the processes that occur during the hydrolytic polycondensation of TEOS.

It should be noted that all of our systems were polydisperse. They had bimodal distributions, reflecting the coexistence of primary particles formed during the polycondensation of tetraethoxysilane, and of secondary aggregates that constituted most of the structural units of the gel. When using silica gel without dopants, polycondensation at the first stage begins with the formation of small nucleus particles, and they then grow. These particles are unstable, however, and they shrink, probably because of hydrolysis. Further interaction between the particles at low $\mathrm{pH}$ values leads to the formation of larger secondary aggregates, which grow because of parallel-forming small particles. These data are in agreement with those obtained by the authors of [13] and are described by the particle growth model proposed in [14].

The introduction of metal cations probably has a stabilizing effect on the polycondensation in a sol-gel system. As a rule, smaller particles form at the first stage, compared to silica gel without dopants. Larger secondary particles then form because of aggregation, as in the case of pure silica gel. Figure 2 shows the growth dynamics of these secondary particles during the hydrolytic polycondensation of tetraethoxysilane in the presence of metal cations.

The gels doped with $\mathrm{Na}^{+}, \mathrm{Ni}^{2+}, \mathrm{Co}^{2+}$, and $\mathrm{Ce}^{3+}$ cations produced bimodal distributions of particles according to volume at the 30th minute of the experiment. The first maximum corresponds to particles with an average hydrodynamic diameter of $1.4 \mathrm{~nm}$; the second, to larger particles with an average hydrodynamic diameter of $\sim 4 \mathrm{~nm}$.

Secondary aggregates formed later for the gels doped with $\mathrm{Ag}^{+}$and $\mathrm{Fe}^{3+}$ cations: they appeared $\sim 8 \mathrm{~h}$ after the start of the reaction. Particles with an average diameter of $15.3 \mathrm{~nm}$ occupied $8 \%$ of the total volume of the system containing silver cations, while smaller particles with a diameter of $\sim 5.4 \mathrm{~nm}$ occupied the remaining volume. Large particles with a diameter of $16.2 \mathrm{~nm}$ occupied $14.6 \%$ after $2 \mathrm{~h}$. The system was homogeneous $12 \mathrm{~h}$ after the start of the reaction, and the probable hydrodynamic particle size was $18.1 \mathrm{~nm}$, which corresponds to the largest particles of all the investigated samples.

Additional data on the structural parameters of the final products of polycondensation-the gels doped with metal cations-were obtained by means of SAXS. Radius of gyration $R_{\mathrm{g}}$ was found from the slope of a linear extrapolation of the initial part of a SAXS curve (the Guinier region) constructed as the relationship
Table 1. Calculated activation energies $E_{\mathrm{a}}$ (in $\mathrm{kJ} / \mathrm{mol}$ ) for different gels

\begin{tabular}{c|c|c|c|c|c|c|c}
\hline $\begin{array}{c}\text { Doping } \\
\text { cation }\end{array}$ & - & $\mathrm{Ag}^{+}$ & $\mathrm{Na}^{+}$ & $\mathrm{Co}^{2+}$ & $\mathrm{Ni}^{2+}$ & $\mathrm{Fe}^{3+}$ & $\mathrm{Ce}^{3+}$ \\
\hline$E_{\mathrm{a}}$ & 49.0 & 64.7 & 57.7 & 63.2 & 40.2 & 44.4 & 54.8 \\
\hline
\end{tabular}

Table 2. Structural parameters of gels, calculated from SAXS data

\begin{tabular}{l|c|c|c|c|c|c|c}
\hline Parameter & - & $\mathrm{Ag}^{+}$ & $\mathrm{Na}^{+}$ & $\mathrm{Co}^{2+}$ & $\mathrm{Ni}^{2+}$ & $\mathrm{Fe}^{3+}$ & $\mathrm{Ce}^{3+}$ \\
\hline$R_{\mathrm{g}}, \mathrm{nm}$ & 9.5 & 8.0 & 9.0 & 9.6 & 7.4 & 7.8 & 7.0 \\
$D_{m}$ & 2.2 & 2.6 & 2.3 & 2.4 & 2.2 & 2.2 & 2.2 \\
\hline
\end{tabular}

between $\ln I$ and $s^{2}$. The fractal dimension was calculated from the tangent of the angle of inclination to the plot of scattering intensity as the relationship between $\log I$ and $\log s$ in the Porod region.

Figure 3 shows typical small-angle scattering curves constructed in the $\log I-\log s$ coordinates, while Table 2 shows the structural parameters calculated from them. It is clear that the introduction of additives of different metal cations affected the structure of the gel. Indeed, the cations (except cobalt) reduced the radius of gyration, compared to silica gel without alloying agents. The smallest radius of gyration was typically seen after the introduction of cerium cations.

The introduction of metal cations limited the total size of fractal aggregates. Our calculations showed that all samples belonged to mass fractals. Fractal dimension $D_{m}$ for all the investigated samples was 2.20 to 2.60, which corresponds to branched structures (Table 2). When $D_{m}$ grew, the gel structure became less

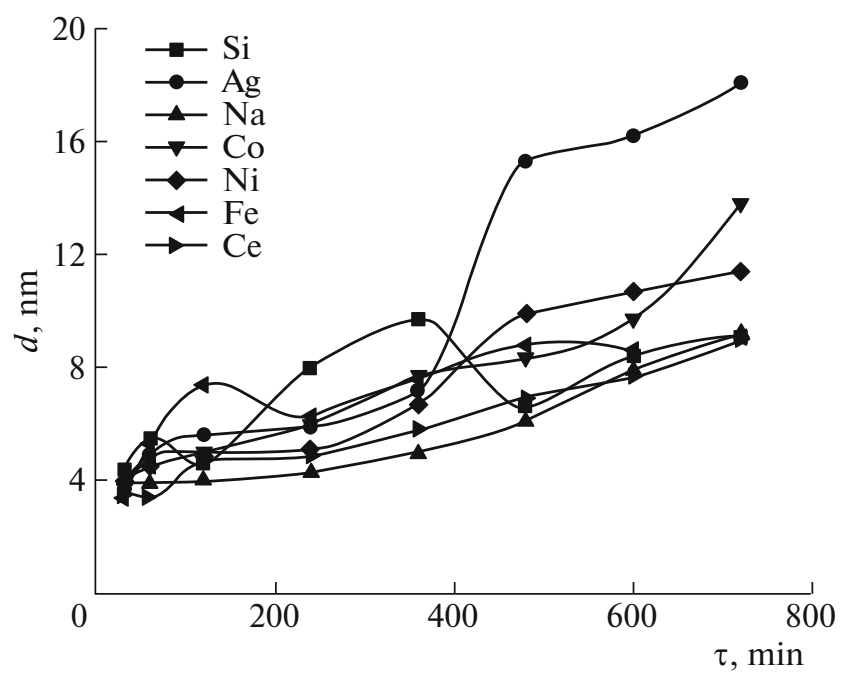

Fig. 2. Change in average hydrodynamic diameter $d$ of secondary aggregates during the hydrolytic polycondensation of TEOS in the presence of various cations. 


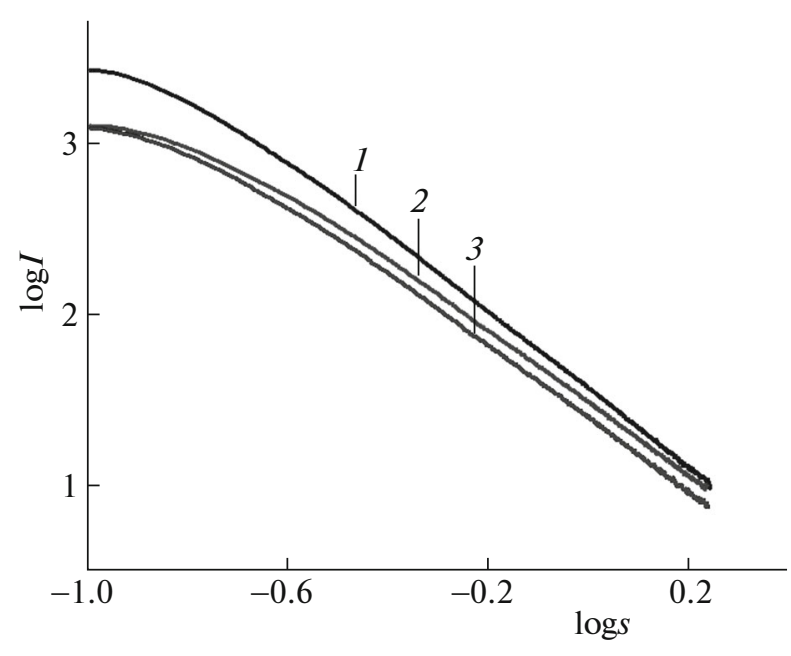

Fig. 3. SAXS curves for silica gel samples doped with cations (1) $\mathrm{Na}^{+}$, (2) $\mathrm{Ce}^{3+}$, and (3) $\mathrm{Fe}^{3+}$.

friable, and a denser cross-linked three-dimensional structure appeared. The gel doped with silver cations had the densest structure among the investigated samples. It is known that when $D_{m}>3$, three-dimensional nets emerge instead of compact particles with rough surfaces; however, no such values were observed for the investigated samples.

We identified a relationship between the fractal dimension and the model of fractal aggregate formation [15]. Two models stand out from among those proposed to describe the aggregation mechanisms in $\mathrm{SiO}_{2}$ systems: diffusion controllable aggregation (DCA, the Witten-Sander model) and kinetically controllable aggregation (KCA) of a cluster-cluster or particle-cluster type. These models produce two structurally different fractal aggregates. Aggregate fractal dimension $D_{m}$ is no more than 2 for the DCA model. The fractal dimension for KCA is $2<D_{m}<3$. The KCA model is characteristic of systems in which there is a high repulsive barrier between particles. Aggregation in this case depends on the potential of interparticle interaction [15]. In this work, the mass fractal dimensions of the aggregates of all the investigated samples corresponded to those formed during kinetically controllable aggregation of the clustercluster type.

We may therefore assume that in interacting with silica-silica particles formed during the hydrolytic polycondensation of tetraethoxysilane, some metal cations (e.g., $\mathrm{Ag}^{+}, \mathrm{Na}^{+}, \mathrm{Co}^{2+}$, and $\mathrm{Ce}^{3+}$ ) raise the interparticle potential to prevent condensation and the formation of gel. Other cations (e.g., $\mathrm{Ni}^{2+}$ and $\mathrm{Fe}^{3+}$ ) probably have no such effect.

\section{CONCLUSIONS}

The addition of doping metal cations has a substantial effect on the kinetics of the formation of tetraethoxysilane gels during hydrolytic polycondensation in acidic environments. We found that introducing metal cations into a tetraethoxysilane system at $40^{\circ} \mathrm{C}$ lengthens the period of gelation time in the series $\mathrm{Ni}^{2+}<$ silica gel without dopants $<\mathrm{Ce}^{3+}<\mathrm{Ag}^{+}<$ $\mathrm{Fe}^{3+}<\mathrm{Co}^{2+}<\mathrm{Na}^{+}$. Introducing metal cations into the system affects the activation energy of gelation: $\mathrm{Fe}^{3+}$ and $\mathrm{Ni}^{2+}$ cations reduce it relative to silica gel without additives, while other cations (especially $\mathrm{Co}^{2+}$ ) increase it considerably. The gels obtained during the hydrolytic polycondensation of tetraethoxysilane in the presence of doping metal cations were mass fractal aggregates. The dimensions of mass fractals show that the mechanism of their formation was kinetically controllable aggregation of the cluster-cluster type.

\section{REFERENCES}

1. T. V. Kon'kova, M. G. Gordienko, M. B. Alekhina, and N. V. Men'shutina, Russ. J. Phys. Chem. A 91, 468 (2017).

2. I. Y. Bu, Optik 162, 121 (2018).

3. Z. Maa and Y. Jiang, Appl. Surf. Sci. 436, 732 (2018).

4. T. F. Kuznetsova, A. I. Rat'ko, and S. I. Eremenko, Russ. J. Phys. Chem. A 86, 1618 (2012).

5. Q. Zhanga and X. Denga, Eng. Failure Anal. 82, 64 (2017).

6. S. Komarneni, I. R. Abothu, and A. V. P. Rao, J. SolGel Sci. Technol., No. 15, 263 (1999).

7. G. R. Lee and J. A. Crayston, J. Adv. Mater. 5, 434 (1993).

8. D. Levy and L. Esquivias, J. Adv. Mater. 7, 120 (1995).

9. A. Yousif, O.-Y. Omer Alhussein, and M. S. Eltoum, Int. J. Multidisc. Sci. Eng. 6, 19 (2015).

10. D. Nagao and H. Osuzu, J. Colloid Interface Sci. 279, 143 (2004).

11. J. Cihlz, Colloids Surf., A 70, 239 (1993).

12. R. K. Iler, The Chemistry of Silica: Solubility, Polymerization, Colloid and Surface Properties and Biochemistry of Silica (Wiley, New York, 1979).

13. B. K. Goutam and D. Ganguli, J. Mater. Chem. 10, 2289 (2000).

14. V. K. Lamer and R. H. Dinegar, J. Am. Chem. Soc. 72, 4847 (1950).

15. V. I. Roldugin, Russ. Chem. Rev. 72, 823 (2003).

Translated by A. Tulyabaev 